\title{
Experimental Method of Temperature and Strain Discrimination in Polymer Composite Material by Embedded Fiber-Optic Sensors Based on Femtosecond-Inscribed FBGs
}

\author{
Victor V. Shishkin, ${ }^{1,2}$ Vadim S. Terentyev, ${ }^{1}$ Denis S. Kharenko, ${ }^{1,2}$ \\ Alexandr V. Dostovalov, ${ }^{1}$ Alexey A. Wolf, ${ }^{1}$ Victor A. Simonov, ${ }^{1}$ Mikhail Yu. Fedotov, ${ }^{3}$ \\ Anton M. Shienok, ${ }^{3}$ Ivan S. Shelemba, ${ }^{2}$ and Sergey A. Babin ${ }^{1}$ \\ ${ }^{1}$ Institute of Automation and Electrometry, Siberian Branch of the Russian Academy of Sciences, \\ 1 Academician Koptug Avenue, Novosibirsk 630090, Russia \\ ${ }^{2}$ Inversion Sensor Co., Ltd., 10625 Oktyabrya Street, Perm 614990, Russia \\ ${ }^{3}$ All-Russian Scientific Research Institute of Aviation Materials, 17 Radio Street, Moscow 105005, Russia \\ Correspondence should be addressed to Victor V. Shishkin; shishkin@i-sensor.ru
}

Received 18 November 2015; Revised 11 March 2016; Accepted 7 April 2016

Academic Editor: Carlos Marques

Copyright (C) 2016 Victor V. Shishkin et al. This is an open access article distributed under the Creative Commons Attribution License, which permits unrestricted use, distribution, and reproduction in any medium, provided the original work is properly cited.

Experimental method of temperature and strain discrimination with fiber Bragg gratings (FBGs) sensors embedded in carbon fiber-reinforced plastic is proposed. The method is based on two-fiber technique, when two FBGs inscribed in different fibers with different sensitivities to strain and/or temperature are placed close to each other and act as a single sensing element. The nonlinear polynomial approximation of Bragg wavelength shift as a function of temperature and strain is presented for this method. The FBGs were inscribed with femtosecond laser by point-by-point inscription technique through polymer cladding of the fiber. The comparison of linear and nonlinear approximation accuracies for array of embedded sensors is performed. It is shown that the use of nonlinear approximation gives 1.5-2 times better accuracy. The obtained accuracies of temperature and strain measurements are $2.6-3.8^{\circ} \mathrm{C}$ and $50-83 \mu \varepsilon$ in temperature and strain range of $30-120^{\circ} \mathrm{C}$ and $0-400 \mu \varepsilon$, respectively.

\section{Introduction}

Polymer composite materials (PCM) are widely used in aerospace industry, shipbuilding, and wind-power energetics due to their lightweight, strength, corrosion immunity, and fire resistance $[1,2]$. One of the key questions for PCM construction details is their long-term reliability. That is why their condition should be monitored in real time to detect damage, delamination, wear, and tear (during fabrication, transportation, and exploitation). Sensors based on fiber Bragg gratings are ideally suited for embedding into composite materials since the optical fiber size is comparable with thickness of composite monolayer. In addition, they enable easy multiplexing and high measurement accuracy with immunity to electromagnetic interference.
Main problem of embedded FBG sensors concerns their simultaneous sensitivity to temperature and strain. Current techniques of their discrimination lead to significant increase in cost and complexity of interrogation equipment. Therefore simpler techniques are required, which will combine advantages of fiber sensors and applicability for embedding into composite materials. One of the most applicable techniques of strain and temperature discrimination in PCM is the method that employs FBGs in two different fibers, embedded side by side [3]. The advantage of the method is that there is no need for fiber etching or polishing [4], splicing [5], or other structural impacts $[6,7]$, which can increase the fiber fragility. On the other hand, it is difficult to find a pair of optical fibers, which could let one achieve the typical figures of merit (sensitivity and repeatability). The method requires 
the temperature and/or strain sensitivity of FBGs, formed in two fibers, to be strongly different from each other [3]. This difference could be achieved with pair of polymer and silica optical fibers, as FBGs written in polymer fibers have negative sign of temperature sensitivity comparing to those in silica fibers [8]. Polymer fibers are commercially available, but they can be damaged during the PCM molding process because of high temperature of annealing or composite curing processes. Core and cladding materials, dopants, and their concentrations are the valuable parameters affecting the temperature sensitivity of the FBG. For example, FBGs in lead-doped fibers have increased sensitivity [9], while gratings in boron doped fibers have decreased one [5]. However, fibers with special dopants have high cost as they are less demanded, comparing to telecommunication fibers like SMF-28e. One more way is to use different types of ultraviolet (UV) inscribed gratings [10], but, in this case, the difference between temperature sensitivities is not big enough. More accessible and cheap variant of UV-inscription methods needs FBG to be recoated with polymer coating after the inscription that may lead to increased fragility in PCM after embedding. In this work femtosecond (fs) inscribed FBGs are used. Advantages of fs-FBGs as compared with UVFBGs consist of the possibility of FBGs inscription through the polymer cladding without damaging cladding $[11,12]$ with increased speed of fabrication. The main disadvantage is the higher scattering due to noncircular shape of fsmodified area in fiber-core cross section. It leads to increased coupling to cladding modes and additional spectral sideband that may influence dynamics of the FBG spectra. Therefore, considering unexpected effects after embedding of fs-FBGs, the method of strain and temperature discrimination based on nonlinear polynomial approximation is used. The method can be useful in other applications, where nonlinear polynomial approximation is necessary to increase the accuracy of measurements.

\section{Methodology}

To separate temperature and strain contributions, the method based on FBGs in two fibers with different characteristics is applied [3]. Two FBGs inscribed in different fibers are located in one measurement point thus producing one sensor. The larger the sensitivity difference between FBGs, the higher the measurement accuracy of the sensor. A polynomial approximation (second-order polynomial for temperature [13] and linear for strain) is introduced, which fits the shift of reflection spectrum $\Delta \lambda_{1,2}$ of FBGs better than linear approximation:

$$
\begin{aligned}
& \Delta \lambda_{1}=K_{1 \varepsilon 1} \varepsilon+K_{1 T 1} \Delta T+K_{2 T 1} \Delta T^{2} \\
& \Delta \lambda_{2}=K_{1 \varepsilon 2} \varepsilon+K_{1 T 2} \Delta T+K_{2 T 2} \Delta T^{2},
\end{aligned}
$$

where $\Delta T=T_{1}-T_{0}$ and $T_{1}$ and $T_{0}$ are current and initial temperatures of the sample. To solve system (1) at $\Delta T$, $\varepsilon$, coefficients of approximating polynomials $K_{1 \varepsilon 1,2}, K_{1 T 1,2}$, and $K_{2 T 1,2}$, must be obtained from a calibration procedure, when the PCM sample is exposed to longitudinal strain load and to variable temperature, while the Bragg wavelengths shifts are measured. Effective coefficients $\bar{K}_{1 \varepsilon 1,2}, \bar{K}_{1 T 1,2}$, and $\bar{K}_{2 T 1,2}$ together with root-mean-square error for wavelength shifts $\delta \lambda_{\mathrm{RMS1,2}}$ of each FBG can be found using least-square method [14]. All possible noises of calibration setup including interrogation system, extensometer, and thermometer are included in $\delta \lambda_{\mathrm{RMS1,2}}$. Another error source is the apparatus error $\delta \lambda_{A}$ of measuring interrogation system (different from calibration one). It can introduce significant errors if fast wavelength meter with comparatively low resolution $(\sim 10-$ $100 \mathrm{pm})$ is used. The apparatus error is included in full wavelength shift error $\delta \lambda_{1,2}$ that can be found as $\delta \lambda_{1,2}^{2} \equiv$ $\delta\left(\Delta \lambda_{1,2}\right)^{2}=\delta \lambda_{\mathrm{RMS} 1,2}^{2}+\delta \lambda_{A 1,2}^{2}$, where $\delta \lambda_{\mathrm{RMS} 1,2}$ and $\delta \lambda_{A 1,2}$ are independent values and normally distributed [15]. Using the notations of temperature error as $\delta T=\delta(\Delta T)$ and strain error as $\delta \varepsilon$, the measurement accuracies of system (1) are estimated as

$$
\begin{aligned}
& \delta T= \pm \frac{\left[\bar{K}_{1 \varepsilon 2}{ }^{2} \delta \lambda_{1}^{2}+\bar{K}_{1 \varepsilon 1}{ }^{2} \delta \lambda_{2}^{2}\right]^{1 / 2}}{\left(\bar{K}_{1 T 1}+2 \bar{K}_{2 T 1} \Delta T\right) \bar{K}_{1 \varepsilon 2}-\left(\bar{K}_{1 T 2}+2 \bar{K}_{2 T 2} \Delta T\right) \bar{K}_{1 \varepsilon 1}} \\
& \delta \varepsilon \\
& = \pm \frac{\left[\left(\bar{K}_{1 T 2}+2 \bar{K}_{2 T 2} \Delta T\right)^{2} \delta \lambda_{1}^{2}+\left(\bar{K}_{1 T 1}+2 \bar{K}_{2 T 1} \Delta T\right)^{2} \delta \lambda_{2}^{2}\right]^{1 / 2}}{\left(\bar{K}_{1 T 1}+2 \bar{K}_{2 T 1} \Delta T\right) \bar{K}_{1 \varepsilon 2}-\left(\bar{K}_{1 T 2}+2 \bar{K}_{2 T 2} \Delta T\right) \bar{K}_{1 \varepsilon 1}} .
\end{aligned}
$$

Equations (2) can be deduced from (1) as errors of indirect measurements. The errors of parameters in mathematical interpretation can be considered as their variations. The differential $(\delta)$ of (1) at $\Delta T, \varepsilon$, and $\Delta \lambda_{1,2}$ shows the influence of this variation on solution of (1). The linear equations system of variations can be solved at variables $\delta T$ and $\delta \varepsilon$. The solution of the second-order linear equation system consists of two terms, $C_{1} \delta \lambda_{1}+C_{2} \delta \lambda_{2}$, where $C_{1,2}$ depend in general on $\Delta T$, $\varepsilon$, and $\bar{K}$-coefficients. As errors $\delta \lambda_{1,2}$ are independent and normally distributed, the $\delta T$ and $\delta \varepsilon$ errors can be estimated from the solution as $\left[\left(C_{1} \delta \lambda_{1}\right)^{2}+\left(C_{2} \delta \lambda_{2}\right)^{2}\right]^{1 / 2}$.

To test the method the following pair of fibers was chosen. Germanium doped fiber Fibercore SM1500P (9/125) (SM1500P-125/150) with diameter $150 \mu \mathrm{m}$ of polyimide coating was used as a fiber with high temperature sensitivity. Boron doped fiber Fibercore PS1250-1500 (PS1250-125/250) with diameter $250 \mu \mathrm{m}$ of acrylate coating was used as a fiber with low temperature sensitivity.

Three FBGs were inscribed in each of these fibers by a femtosecond laser with the wavelength of $1026 \mathrm{~nm}$. The femtosecond inscription was performed by point-by-point technique with femtosecond laser through the coating [12]; therefore mechanical characteristics of fibers did not change. It is known that the temperature sensitivities for FBGs inscribed by UV methods in fibers PS1250 and SM1500P are different and approach about 9 and $13 \mathrm{pm} /{ }^{\circ} \mathrm{C}$ correspondingly [5]. This difference was supposed to be enough for effective discrimination of temperature and strain with estimated accuracy not worse than $10^{\circ} \mathrm{C}$ and $100 \mu \varepsilon$ correspondingly with linear approximation and apparatus error of wavelength meter about $20 \mathrm{pm}$ (fast device with comparatively low 


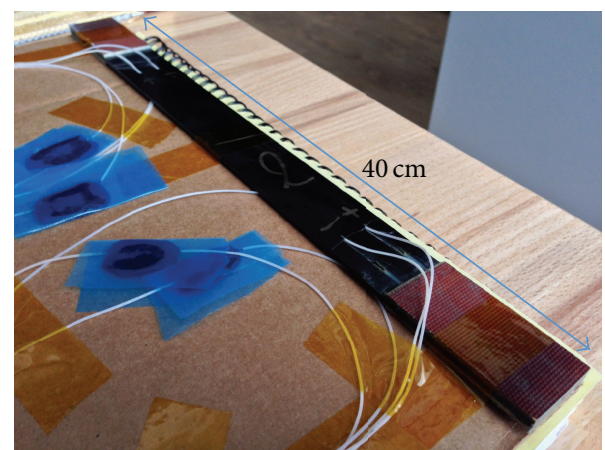

FIGURE 1: The CFRP sample with embedded fiber-optic sensors.

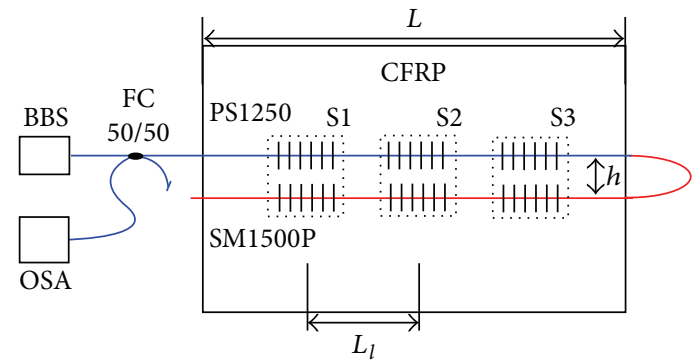

Figure 2: The topology of the fiber sensors in the sample, S1, S2, and S3 (FBG sensor array).

resolution for impact control). We believe that it is possible to improve the accuracy using the nonlinear approximation.

The packages of prepreg were assembled for experimental tests. They were based on epoxy fusion adhesive VSE-1212 and medium-tensile carbon fiber Toho Tenax IMS65 of 500 $\times 500 \times 2 \mathrm{~mm}$ size with layer reinforcing structure $[0]_{n}$ and formed slabs, from which the samples of carbon fiberreinforced plastic $(\mathrm{CFRP}) 400 \times 25 \times 2 \mathrm{~mm}$ size have been fabricated by mechanical cutting [16]. Two CFRP samples were fabricated (Figure 1 shows number 2). Two fibers, each comprising three FBGs of $2 \mathrm{~mm}$ length separated by $L_{1}=$ $40 \mathrm{~mm}$, were placed close $(h=1 \mathrm{~mm})$ to each other between central prepreg layers in parallel with the long edge of sample in such a way that the FBG sensor array (S1, S2, and S3) was located in the central area of samples (Figure 2). Optical fibers were routed out of the sample surface at angle of $15^{\circ}$ through PTFE tubes safely securing the fiber with a sufficient flexibility. Molding of prepreg's packages was made in autoclave with maximum temperature $180^{\circ} \mathrm{C}$ and specific pressure not exciding $0.7 \mathrm{MPa}$.

\section{Experiment and Results}

Figure 3 shows the measured spectra of the fiber sensors embedded into the CFRP number 2 sample (line) and not embedded (dotted line). Normalized spectra show slightly decreasing amplitude of peaks for PS1250 FBGs after embedding. This is possible due to microbending of fiber and increasing of scattering. Since embedding into the sample does not influence sufficiently the spectra, it seems possible

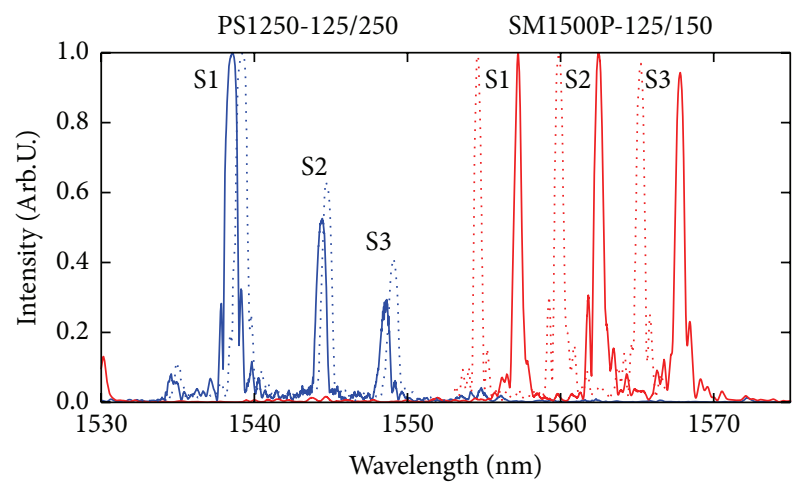

FIGURE 3: Normalized reflection spectra of FBGs before (dotted line) and after (line) the embedding into CFRP.

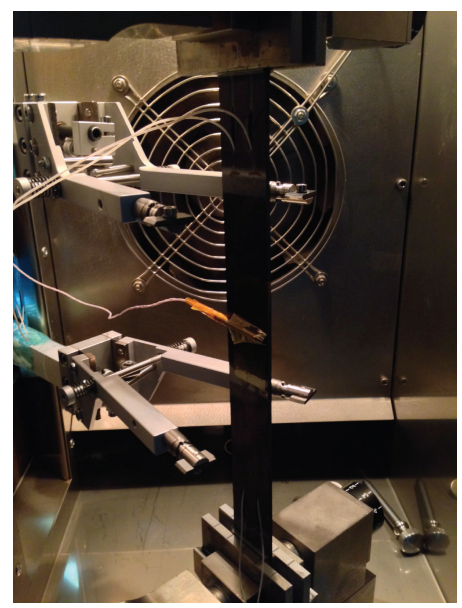

FIgURE 4: A sample of CFRP in testing machine.

to use standard interrogation techniques and algorithms for identification of spectral peak position. The FBGs in SM1500P fiber (red) have smaller losses than those for FBGs in PS1250 fiber (blue), which probably have scattering nature since the intensity decreases from peak to peak. The absolute peak reflections of FBGs are about $60 \%$ for SM1500P and $40-$ 90\% for PS1250.

In the next step, coefficients of temperature and strain sensitivity for FBGs embedded into the CFRP sample were determined with testing machine Zwick/Roell Z250 comprising temperature chamber and extensometer (Figure 4). During the test, the sample was heated up to $+30,+55,+80$, +100 , and $+120^{\circ} \mathrm{C}$. One cycle of loading unloading to $2.5 \mathrm{kN}$ with a step of $0.5 \mathrm{kN}$ was performed at each temperature step (Figure 5(a)). The load value was limited by the grips used. Peak wavelength shifts were measured by means of interrogator Astro A322 with resolution of $1 \mathrm{pm}$ at $1 \mathrm{~Hz}$ sampling rate. Sample elongation was measured by extensometer with $\sim 1$ micron accuracy (declared). The measured longitudinal strain was $400 \mu \varepsilon$. Temperature was measured by thermoresistor fixed at CFRP sample with accuracy of $0.04^{\circ} \mathrm{C}$.

Figure 5(a) shows that the FBGs in PS1250-125/250 fiber have higher temperature sensitivity than that for FBGs in SM1500P-125/150 fiber. The effect of PS1250-125/250 


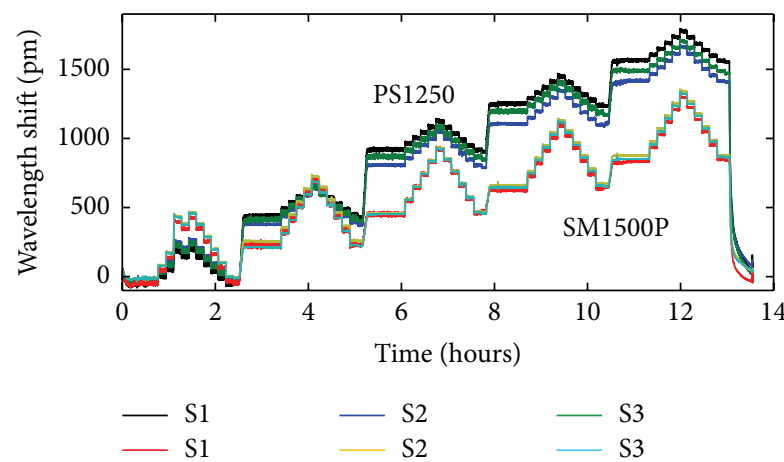

(a)

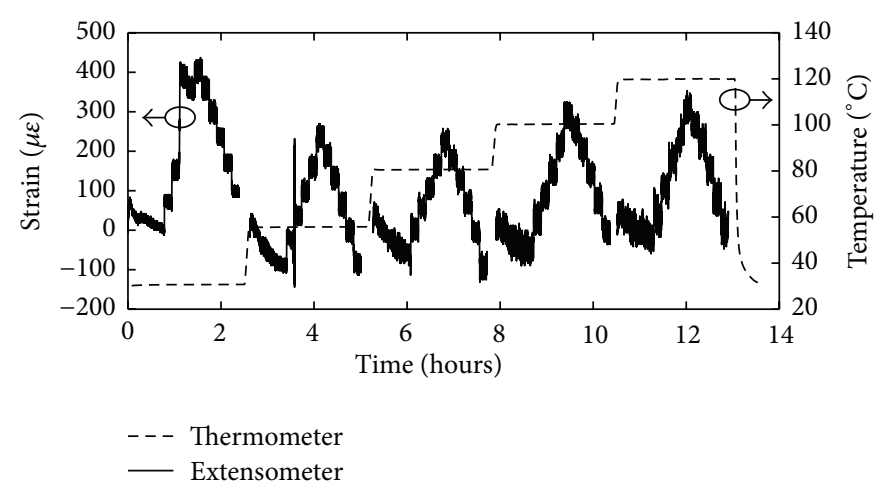

(b)

FIgURE 5: Experiment data. (a) The wavelength shifts of FBGs, (b) strain (line), and temperature (dashed line) during calibration process.

TABLE 1

\begin{tabular}{lccccccc}
\hline Fiber & Sensor & Wavelength, $\mathrm{nm}$ & $\bar{K}_{1 \varepsilon}, \mathrm{pm} / \mu \varepsilon$ & $\bar{K}_{1 T}, \mathrm{pm} /{ }^{\circ} \mathrm{C}$ & $\bar{K}_{2 T}, \mathrm{pm} /{ }^{\circ} \mathrm{C}^{2}$ & $\delta \lambda_{\mathrm{RMS}}, \mathrm{pm}$ & $\delta \lambda_{\mathrm{RMS}}^{(\mathrm{lin})}, \mathrm{pm}$ \\
\hline \multirow{3}{*}{ PS1250 } & $\mathrm{S} 1$ & 1538.56 & 0.528 & 22.07 & $-5.02 e-05$ & 29.2 \\
& $\mathrm{~S} 2$ & 1544.40 & 0.638 & 18.65 & $-3.22 e-05$ & 25.9 & 58.7 \\
& $\mathrm{~S} 3$ & 1548.62 & 0.540 & 20.22 & $-3.81 e-05$ & 31.7 & 50.7 \\
\hline \multirow{3}{*}{ SM1500P } & $\mathrm{S} 1$ & 1557.13 & 1.225 & 16.50 & $-7.09 e-05$ & 50.5 & 87.9 \\
& $\mathrm{~S} 2$ & 1562.43 & 1.232 & 15.64 & $-6.21 e-05$ & 51.6 & 81.4 \\
& $\mathrm{~S} 3$ & 1567.75 & 1.212 & 14.98 & $-5.47 e-05$ & 45.1 & 71.5 \\
\hline
\end{tabular}

increased thermal sensitivity may be explained by influencing of some factors and/or their combinations. Probably, the main factor is different influence of embedding process on different types of fibers, especially on fiber coatings. The less probable factor is the influence of scattering in fs-FBG and microbending of fibers inside CFRP on the dynamics of FBGs spectra. The picture also shows that FBGs in SM1500P-125/150 fiber have higher strain sensitivity. It may be explained by better adhesion of polyimide coating to polymer matrix of the sample. Figure 5(b) shows data from thermometer and extensometer corresponding to Figure 5(a). There was high noise of extensometer, which makes noticeable contribution to main calibration errors $\delta \lambda_{\mathrm{RMS1,2}}$. The measured sensitivity coefficients are presented in Table 1.

Calibration errors $\delta \lambda_{\mathrm{RMS}}^{(\mathrm{lin})}$ for linear approximation are shown in the last column of Table 1 , which are 1.5-2 greater than the second-order ones, $\delta \lambda_{\text {RMS }}$.

According to formulae (2) in case of the nonlinear polynomial approximation, errors depend on the absolute values of temperature and strain. It means that in general case the errors need to be represented in 3D or in contour graphics. As (2) are independent of absolute strain, Figure 6 demonstrates (in 2D) the accuracy of sensors consisting of two FBGs in PS1250 and SM1500P fibers: pairs are S1 (1538.56, 1557.13), S2 (1544.40, 1562.43), and S3 (1548.62, 1567.75), with the above-mentioned values of coefficients and apparatus error $\delta \lambda_{A}=10 \mathrm{pm}$. Presented errors for temperature (Figure 6(a)) and strain (Figure 6(b)) are smaller for the second-order approximation than for linear one in whole temperature interval. The accuracy value varies in $2.6-3.8^{\circ} \mathrm{C}$ range for temperature and 50-83 $\mu \varepsilon$ for strain. Probably, the calibration accuracy is limited by the extensometer noise (Figure 5(b)), as it does not provide enough precision at low strain level.

As follows from Table 1, thermal sensitivities $\bar{K}_{1 T}$ for FBG in PS1250 fiber embedded in the composite exceed standard values two times for bare fiber. To prove the influence of embedding on FBG's properties the temperature sensitivity test of bare FBGs in PS1250 fiber was carried out. Three FBGs at a distance $40 \mathrm{~mm}$ between each other were written by the same point-to-point femtosecond laser modification method. The result is $K_{1 T 1}=\{8.5,11.1,11.6\} \mathrm{pm} /{ }^{\circ} \mathrm{C}$, which corresponds to FBGs formed by UV-inscription in the same fiber [5]. The sensitivity of FBGs in SM1500P in bare fiber has increased insignificantly compared to UV FBGs, which typically is $K_{1 T 2} \approx 13 \mathrm{pm} /{ }^{\circ} \mathrm{C}$.

Reproducibility of embedding process is confirmed by almost the same temperature dependence of FBGs wavelength shifts in sample number 1 as in number 2 without tensile force. Full calibration procedure with sample number 1 was not performed.

\section{Conclusion}

The experiment shows that the proposed experimental method of temperature and strain discrimination is applicable for its fabrication, calibration, and utilizing of CRFP with embedded fiber-optic fs-FBGs sensors. The nonlinear approximation for wavelength shift is presented, along with analytical formulae for strain and temperature errors. The comparison of linear and nonlinear approximation accuracies was performed. It was shown that the use of nonlinear approximation results in 1.5-2 times better accuracy. The 


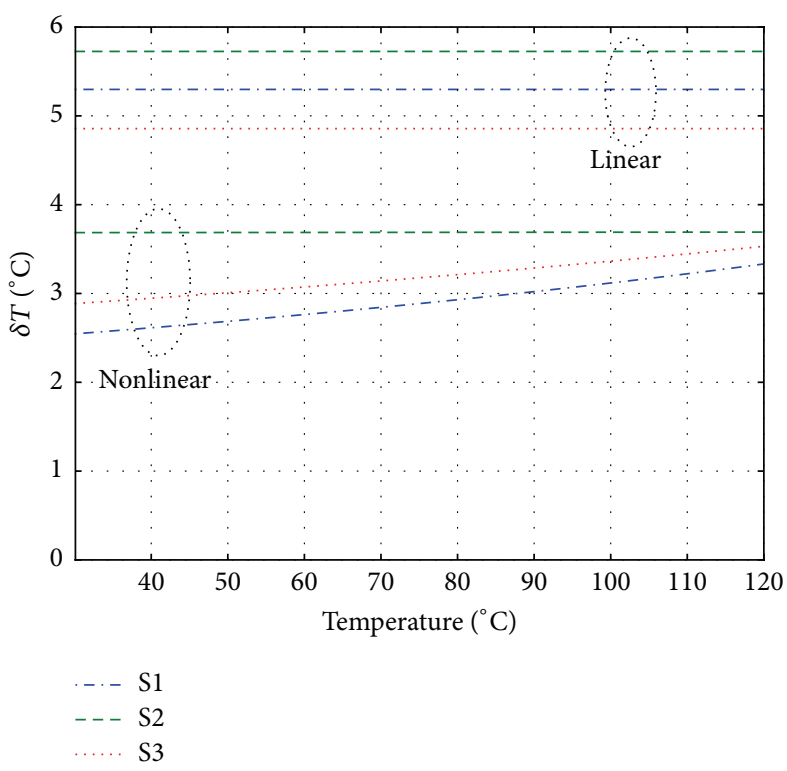

(a)

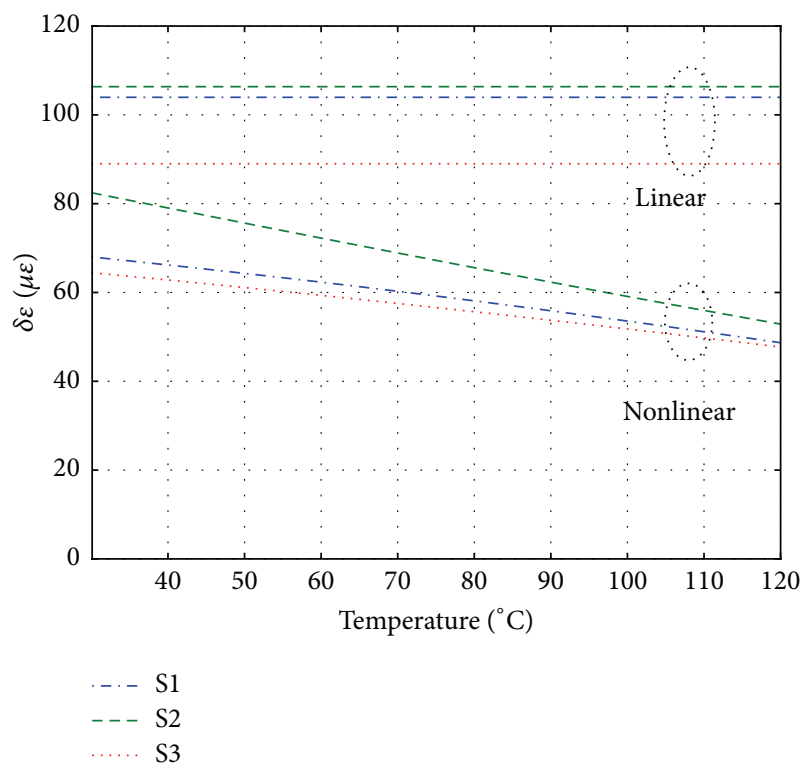

(b)

Figure 6: The accuracy $\delta T$ of temperature (a) and $\delta \varepsilon$ of strain (b) separation versus temperature $T_{1}$. Dotted circles show corresponding approximation polynomial.

obtained accuracies of temperature and strain measurements are $2.6-3.8^{\circ} \mathrm{C}$ and $50-83 \mu \varepsilon$ in temperature and strain range of $30-120^{\circ} \mathrm{C}$ and $0-400 \mu \varepsilon$, respectively.

\section{Competing Interests}

The authors declare that they have no competing interests.

\section{References}

[1] R. Di Sante, "Fibre optic sensors for structural health monitoring of aircraft composite structures: recent advances and applications," Sensors, vol. 15, no. 8, pp. 18666-18713, 2015.

[2] W. J. Staszewski, C. Boller, and G. R. Tomlinson, "Health monitoring of aerospace structures," in Smart Sensor Technologies and Signal Processing, p. 266, John Wiley \& Sons, New York, NY, USA, 2004.

[3] P. Sivanesan, J. S. Sirkis, Y. Murata, and S. G. Buckley, "Optimal wavelength pair selection and accuracy analysis of dual fiber grating sensors for simultaneously measuring strain and temperature," Optical Engineering, vol. 41, no. 10, pp. 2456-2463, 2002.

[4] W. Zhou, C.-L. Zhao, J. Huang, and X. Dong, "A novel FBG sensing head geometry for strain-temperature discrimination," in Proceedings of the Asia Communications and Photonics Conference and Exhibition (ACP '09), pp. 1-6, Shanghai, China, November 2009.

[5] H. J. Yoon, D. M. Costantini, H. G. Limberger, R. P. Salathé, C. G. Kim, and V. Michaud, "In situ strain and temperature monitoring of adaptive composite materials," Journal of Intelligent Material Systems and Structures, vol. 17, no. 12, pp. 1059-1067, 2006.

[6] S. F. Silva, O. Frazão, J. L. Santos, L. A. Ferreira, and F. M. Araújo, "Independent strain measurement using a fibre bragg grating written in an array of fused tapers," in Proceedings of the Optical Fiber Sensors Conference (OFS '06), vol. 60, pp. 1-4, October 2006.

[7] O. Frazão, M. Melo, P. V. S. Marques, and J. L. Santos, "Chirped Bragg grating fabricated in fused fibre taper for straintemperature discrimination," Measurement Science and Technology, vol. 16, no. 4, pp. 984-988, 2005.

[8] G. Rajan, M. Ramakrishnan, Y. Semenova, E. Ambikairajah, G. Farrell, and G.-D. Peng, "Experimental study and analysis of a polymer fiber bragg grating embedded in a composite material," Journal of Lightwave Technology, vol. 32, no. 9, pp. 1726-1733, 2014.

[9] S. Ju, P. R. Watekar, and W.-T. Han, "Enhanced sensitivity of the FBG temperature sensor based on the $\mathrm{PbO}-\mathrm{GeO}_{2}-\mathrm{SiO}_{2}$ glass optical fiber," Journal of Lightwave Technology, vol. 28, no. 18, pp. 2697-2700, 2010.

[10] X. Shu, Y. Liu, D. Zhao et al., "Dependence of temperature and strain coefficients on fiber grating type and its application to simultaneous temperature and strain measurement," Optics Letters, vol. 27, no. 9, pp. 701-703, 2002.

[11] J. Thomas, C. Voigtländer, R. G. Becker, D. Richter, A. Tünnermann, and S. Nolte, "Femtosecond pulse written fiber gratings: a new avenue to integrated fiber technology," Laser \& Photonics Reviews, vol. 6, no. 6, pp. 709-723, 2012.

[12] A. A. Wolf, A. V. Dostovalov, and S. A. Babin, "Direct writing of long-period and fiber Bragg gratings in specialty fibers by femtosecond laser pulses at the wavelength of $1026 \mathrm{~nm}$," in Proceedings of the European Conference on Lasers and Electro-OpticsEuropean Quantum Electronics Conference (CLEO/EuropeEQEC '15), paper CM-4.2, Munich, Germany, 2015.

[13] R. Mahakud, J. Kumar, O. Prakash, and S. K. Dixit, "Study of the nonuniform behavior of temperature sensitivity in bare and embedded fiber bragg gratings: experimental results and analysis," Applied Optics, vol. 52, no. 31, pp. 7570-7579, 2013. 
[14] T. Strutz, Data Fitting and Uncertainty: A Practical Introduction to Weighted Least Squares and Beyond, Vieweg, Teubner Verlag, 2011.

[15] J. R. Taylor, An Introduction to Error Analysis the Study of Uncertaintiesin Physical Measurements, University Science Books, 1997.

[16] R. P. Beukema, "Embedding technologies of FBG sensors in composites: technologies, applications and practical use," in Proceedings of the 6th European Workshop on Structural Health Monitoring, vol. 3, Dresden, Germany, July 2012. 


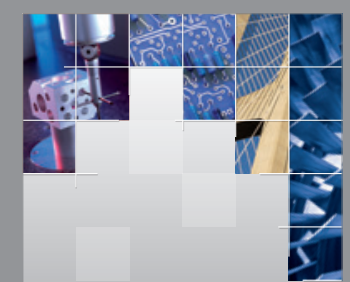

\section{Enfincering}
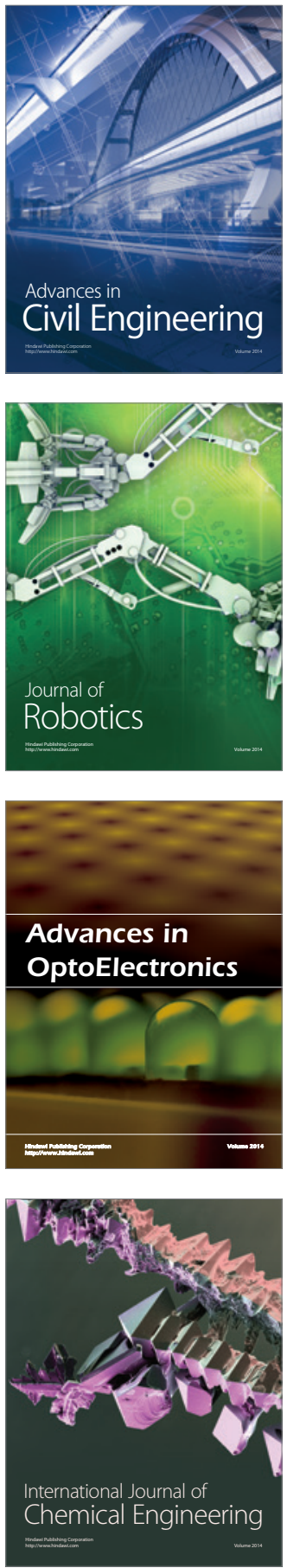

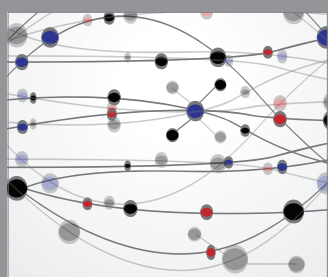

The Scientific World Journal

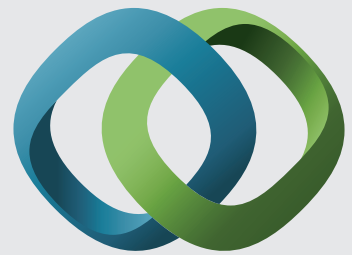

\section{Hindawi}

Submit your manuscripts at

http://www.hindawi.com
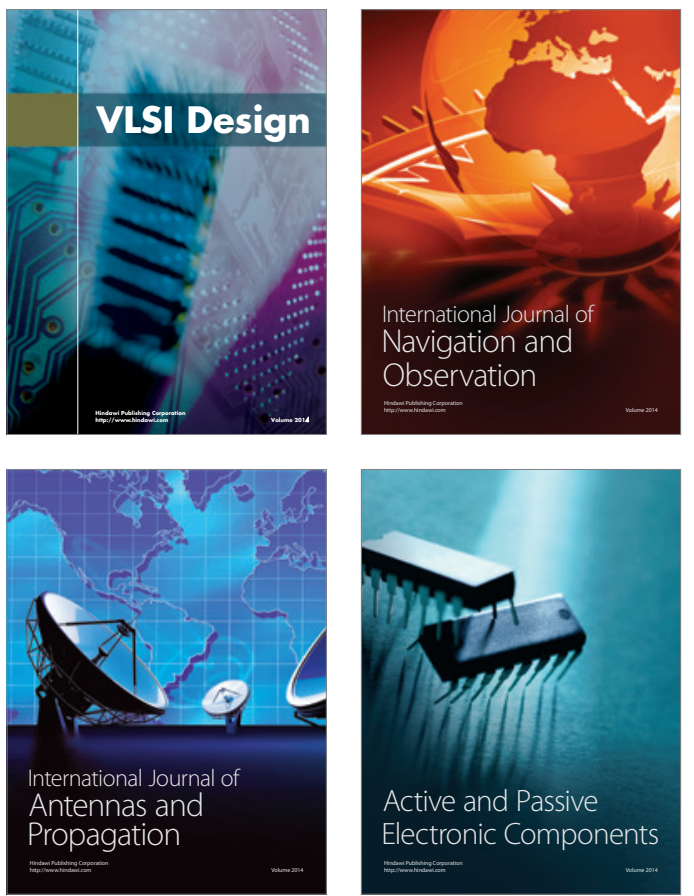
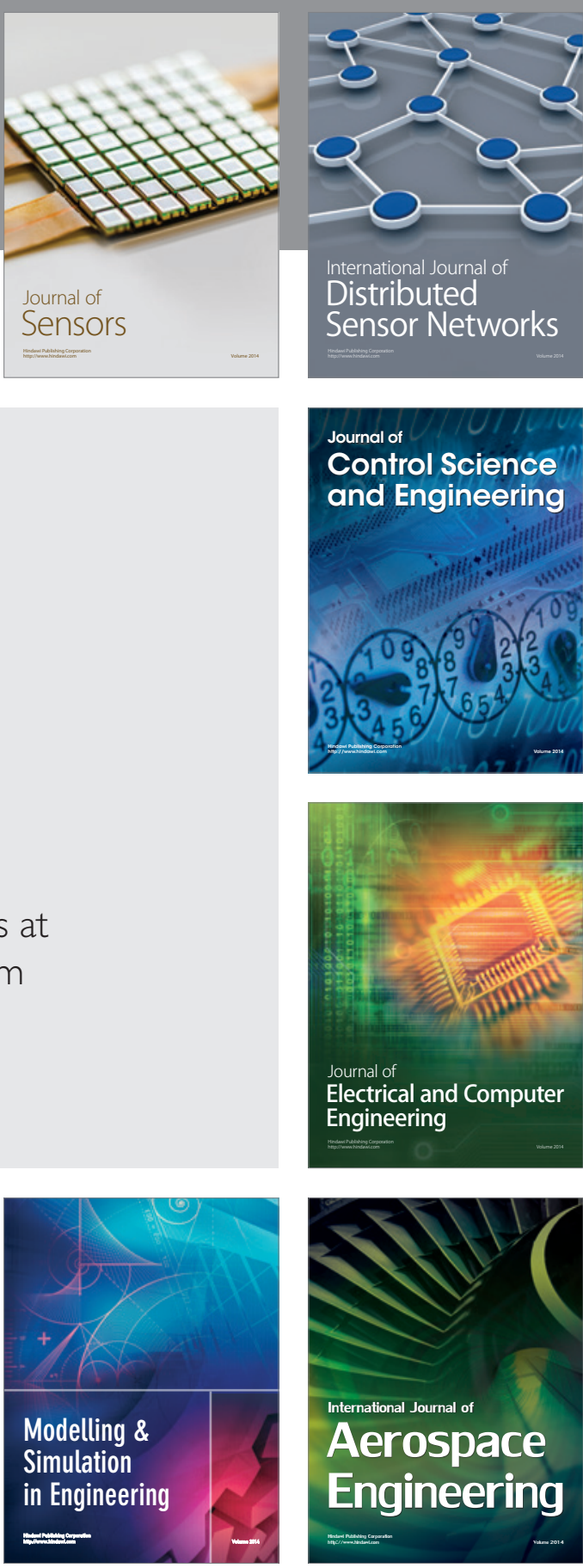

International Journal of

Distributed

Sensor Networks

Journal of

Control Science

and Engineering
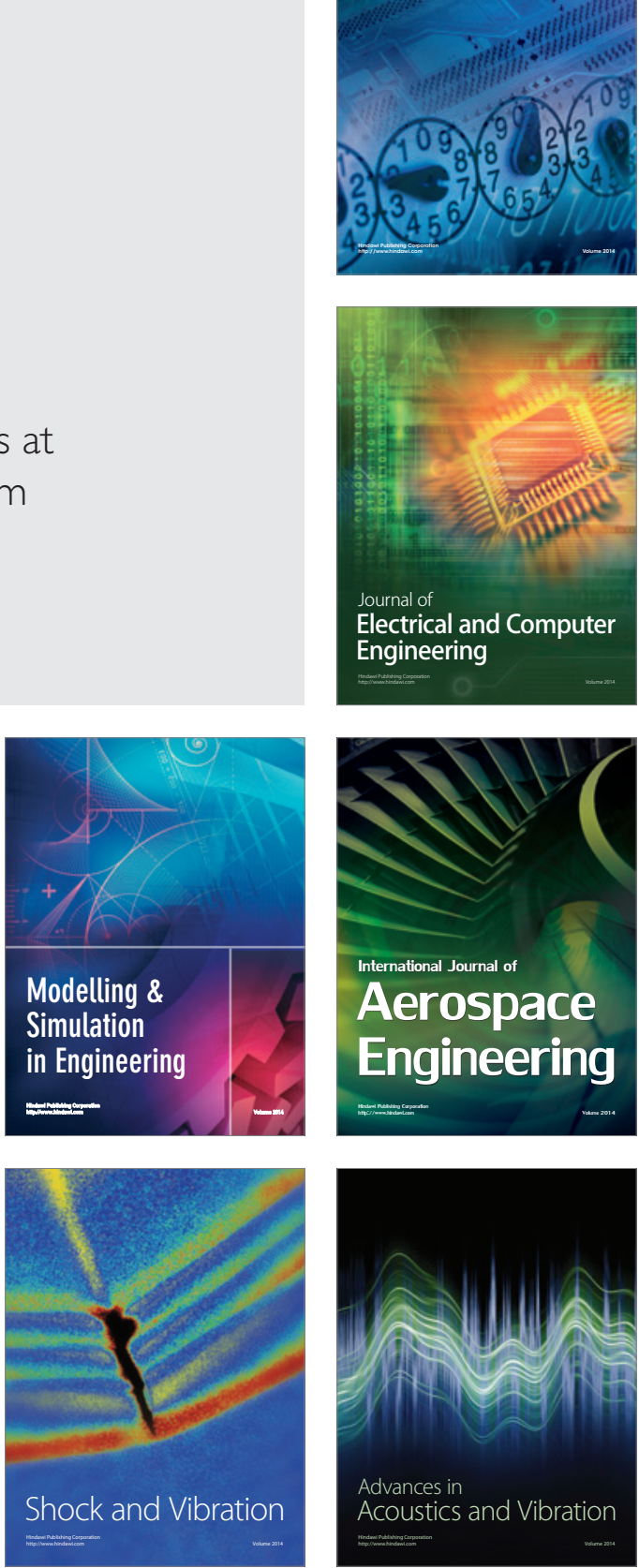